\title{
Strahlenschutz im OP
}

\section{Michael Kraus, Michael Redies, Peter Richter}

\section{Zusammenfassung}

Ionisierende Strahlung ist ein permanentes Risiko für exponiertes Personal. Neben Hauterkrankungen besteht auch das Risiko maligner Entartungen. Durch neue, minimalinvasive OP-Verfahren kommt es zu einer höheren Strahlenemission im OP im Vergleich zu früher. Insgesamt nimmt die Exposition durch medizinische Anwendungen in allen Bereichen $\mathrm{zu}$. Kenntnisse des Strahlenschutzes sind daher essenziell, um sich und andere optimal zu schützen. Diese Übersichtsarbeit soll durch aktuelle Erkenntnisse eigener Studien und Darstellung der Literatur im Umgang mit Röntgenstrahlung sensibilisieren.

\section{Radiation Protection in the Operating Room}

The medical emission of ionising radiation is constantly growing and exposes the OR personnel to a hazardous health risk. Minimally-invasive procedures, as beneficial as they might be for the patient, contribute to this increase of intraoperative radiation in all medical specialities. Therefore it is essential for the people who work with radiation on a daily basis to have an in-depth knowledge of the risks and safety measures. This article aims to give an overview of the current literature and our own data dealing with the issue of radiation in the surgical OR. It is intended to provide the reader with the necessary knowledge to reduce radiation exposition to a minimum.

\section{Einleitung}

Ionisierende Strahlung stellt für beruflich Exponierte eine konstante Gefahrenquelle dar, wobei unter den exponierten Mitarbeitern in medizinischen Bereichen besonders die Kardiologie, die Herz- und Gefäßchirurgie, die Radiologie sowie die Orthopädie und Unfallchirurgie betroffen sind [1]. In einer Fallstudie aus dem Jahr 2005 konnte unter der zuletzt genannten Gruppe auch im Vergleich zu anderen Abteilungen eines Krankenhauses eine signifikante $\mathrm{Zu}$ nahme maligner Erkrankungen gesehen werden [2]. Auch in diesem Journal werden immer wieder, und in den letzten Jahren vermehrt, minimalinvasive Techniken in Orthopädie und Unfallchirurgie dargestellt. Durch eine Schonung des Weichteilmantels und verminderter Ex-

OP-JOURNAL 2014; 30: 138-143

(c) Georg Thieme Verlag KG Stuttgart · New York DOI http://dx.doi.org/10.1055/s-0034-1383302 position des Knochens wurden diverse Vorteile für den Patienten postuliert. Allerdings bedingt eine verminderte Visualisierung des OP-Feldes auch, dass zur Darstellung der Reposition und Implantatplatzierung vermehrt Röntgenstrahlung zum Einsatz kommt [3]. Zusätzlich kommen zunehmend 3-D-basierte Anwendungen zum Einsatz. Dabei werden mithilfe des C-Bogens bis zu 100 Aufnahmen aus verschiedenen Winkeln angefertigt, aus denen ein CT-artiger Datensatz rekonstruiert werden kann. Diese Rekonstrution des Datensatzes kann direkt intraoperativ durchgeführt werden. Patienten, vor allen Dingen aber Mitarbeiter im OP, werden dabei permanent ionisierender Strahlung exponiert. Des Weiteren kommen in Deutschland in aller Regel keine speziell ausgebildeten medizinisch-technischen Radiologieassistenten (MTRA) zum Einsatz, die im Umgang mit mobilen Röntgengeräten geschult sind, sondern Chirurgen und Pflegepersonal, welche die Bildgebung normalerweise selber durchfüh- ren. Daher ist, neben den gesetzlich vorgeschriebenen Weiterbildungen, eine intensive Beschäftigung mit dem Thema unerlässlich, um möglichst wenig Strahlung für eine optimale Bildgebung freizusetzen. Besonders die Grundlagen des Strahlenschutzes und Kenntnisse der Emission von Strahlung durch den Einsatz der mobilen Geräte sind hier erforderlich. Dieser Artikel hat daher zum Ziel, aktuelle Studien zu diesem Thema darzustellen und exponierte Mitarbeiter hinsichtlich der Wichtigkeit der Verminderung der Exposition zu sensibilisieren.

Im chirurgischen OP wird, auch durch die Einführung minimalinvasiver Techniken und Prozeduren, vermehrt Strahlung freigesetzt. Die von der Röntgenverordnung vorgeschriebenen Grenzwerte sind empirisch ermittelt. Ionisierende Strahlung ist ein Gesundheitsrisiko, eine sichere Schwellendosis existiert nicht.

\section{Material und Methoden}

Wir führten eine umfassende Analyse aktuell publizierter Studien durch. Dabei wurde die PubMed-Datenbank zur Recherche verwendet. Die dabei zum Einsatz kommenden Schlüsselwörter waren intra-operative radiation, radiation and orthopaedics und radiation exposure and orthopaedic surgery. Deutsch- und englischsprachige Artikel wurden eingeschlossen. Bei allen identifizierten Artikeln, die zum Thema beitrugen, wurden die jeweiligen Referenzartikel auf weitere Publikationen hin untersucht. Mit diesen Einschlusskriterien konnten insgesamt 30 Artikel gefunden werden, die direkt zum Thema beitragen. Diese Artikel wurden dann einzeln analysiert hinsichtlich ihres Beitrags zur Erlangung relevanter, neuer Erkenntnisse zum Thema dieser Arbeit. 


\section{Ergebnisse}

Wir alle sind stets natürlicher Strahlenbelastung ausgesetzt. Diese können dabei in interne (z. B. durch aufgenommene Speisen, Wasser und vor allen Dingen durch eingeatmetes Radongas) und externe (kosmische Strahlung) Faktoren unterschieden werden. Dabei kommt, je nach Wohnort und Lebensweise, eine jährliche Dosis von ca. 3 Millisievert (mSv) für Mitteleuropäer zusammen. Durch medizinische Anwendungen allein kommen hier noch einmal durchschnittlich 0,6-1,8 mSv hinzu [4]. In Deutschland wird der Umgang mit Strahlenquellen in der Röntgenverordnung (RöV) geregelt. Hier werden auch entsprechend den Hinweisen einiger Fachgremien wie z.B. der International Commission on Radiological Protection (ICRP) Grenzwerte und Richtlinien festgelegt, inwieweit eine berufliche Exposition stattfinden kann [5]. Aktuell gilt für nicht beruflich exponierte Personen ein Grenzwert von $1 \mathrm{mSv} / \mathrm{Jahr}$, exponierte Personen können bis zu 20 mSv/Jahr exponiert werden, wobei diese Dosis über einen 5-Jahres-Zeitraum gemittelt wird. Nicht nur die Körperdosis muss hierbei beachtet werden, auch Organdosen unterliegen gesetzlich geregelten Grenzwerten. Ziel der Ziehung dieser Grenzwerte ist es, Strahlenschäden zu vermeiden. Diese werden in sog. stochastische, also zufällige, und deterministische Strahlenschäden unterschieden [6]. Dabei können durch das Einhalten der Grenzwerte nur deterministische Schäden verhindert werden. Biologisch betrachtet gibt es also keine Schwelle, unter der es unter keinen Umständen zu Strahlenschäden, und seien diese zufällig, kommt [7-10]. Hauptproblem der intraoperativen Durchleuchtung ist dabei die Streustrahlung. Es erreichen insgesamt nur ca. 2\% der Strahlung den Bildverstärker und können somit zur Bilderstellung genutzt werden, der Rest der Strahlung ist Streustrahlung und trägt somit maßgeblich zur Exposition des Patienten und des OP-Personals bei. Der größte Anteil davon wird vom Patienten absorbiert, immerhin noch 10$20 \%$ der Ausgangsstrahlung werden reflektiert oder nicht absorbiert und strahlen somit in die Umgebung ab [11]. Ein großes Problem bei der exakten Bestimmung der Strahlung im OP stellen hierbei die Messverfahren dar. Zur exakten Bestimmung werden dazu Ionisationskammern verwendet. Dies ist in vivo kaum möglich, daher kommen für derartige Studien oftmals Thermolumines- zenzdosimeter (TLD) zum Einsatz. Unter Erhitzung geben diese die absorbierte Strahlung als Licht wieder frei und machen diese somit messbar. In einer Reihe von Untersuchungen wurde dabei versucht, anhand klinischer Szenarien die exakte Exposition des OP-Personals zu detektieren und daraus Vorschläge zu erarbeiten, wie die Dosis reduziert werden kann.

Auf der Seite der Strahlenquelle besteht eine deutlich höhere Exposition als auf der Seite des Bildverstärkers.

Müller et al. verwendeten TLD bei der Stabilisierung von Tibiafrakturen mittels intramedullärer Nagelung an 41 Patienten. Dabei betrug die durchschnittliche Dauer der Strahlenemission 4,6 Minuten. Dabei bekam der Operateur eine Dosis von $1,27 \mathrm{mSv}$ und der 1 . Assistent von $1,19 \mathrm{mSv}$ ab [12]. Bereits 1983 bestimmte Barry mittels TLD-Messung seine eigene Personendosis und zeigte, dass die höchsten Messwerte in der Kopf- und Nackenregion zustande kamen. Hier absorbierte der Autor während eines Jahres in der orthopädischen Chirurgie eine Dosis von umgerechnet $2,27 \mathrm{mSv}$, was innerhalb der zugelassenen Grenzen liegt, jedoch bereits zeigt, dass der Oberkörper in bestimmten Situationen besonders exponiert ist und hier persönliche Schutzmaßnahmen ergriffen werden sollten [13]. Besonderes Augenmerk schenkten 1993 Sanders et al. der Handexposition, da insbesondere in der Traumatologie während der Frakturreposition die Hände besonders betroffen sind. Er ermittelte, dass beide Hände gleichermaßen exponiert werden, unabhängig von der führenden Seite des Operateurs und dass es erst ab einer durchschnittlichen Emission von über 1,7 Minuten zu einer messbaren Belastung kommt [14]. In einer eigenen Untersuchung konnten wir erstmals den Zusammenhang zwischen Frakturschwere, Erfahrung des OP-Teams und der Strahlenemission nachweisen. An knapp 100 konsekutiven Radiusfrakturen zeigte sich nicht nur, dass je komplexer die Fraktur ist, um so mehr Strahlung eingesetzt wird, sondern dass es die Erfahrung des gesamten Teams ist, die einen entscheidenden Einfluss auf die Dauer der Emission nehmen kann. Dabei spielte es keine Rolle, ob der erfahrene Operateur selbst operierte, entscheidend war die reine Präsenz am OP-Tisch [15].

An Studien aus dem Bereich der Wirbelsäulenchirurgie, bei der besonders hohe
Dosen zu erwarten sind [16], konnte der Einfluss der Röntgenröhrenposition auf die Verteilung der Streustrahlung gezeigt werden. So zeigten Rampersaud et al., dass die Dosis am Torso des Chirurgen, insbesondere auf der Seite der Röntgenquelle, immens hoch sein kann. Auf der Seite des Bildverstärkers, also in Strahlungsrichtung, war die Dosis signifikant geringer (53,3 vs. $0,022 \mathrm{mSv} /$ min). Auch die Handdosis konnte durch diese Positionierung deutlich reduziert werden. Die Schilddrüsendosis, die hier mit einer Messkammer ermittelt wurde, war auf der Seite der Röntgenquelle 3-4fach höher als auf der Gegenseite [17]. Nicht nur die laterale Position der Röhre spielt eine entscheidende Rolle, sondern auch die Positionierung in a.-p.-Orientierung. Bei einer Positionierung der Quelle oberhalb des Patienten, im Gegensatz zur Untertischposition, kommen diverse Autoren zu der Erkenntnis, dass zwar eine bessere Arbeitshöhe erreicht werden kann, da die Röhre weniger raumfordernd ist als der Bildempfänger, es dabei jedoch zu einer erhöhten Exposition im Bereich des Oberkörpers, des Kopfes, der Augen und der Schilddrüse kommt $[11,18,19]$. Noch entscheidender ist jedoch die Einhaltung des AbstandsQuadrat-Gesetzes (Abb. 1). In diversen Studien konnte gezeigt werden, dass neben einem vollständigen Verzicht auf Strahlung vor allen Dingen ein großer Abstand zur Quelle den effektivsten Schutz darstellt. So konnte von einer Arbeitsgruppe gezeigt werden, dass bereits durch einen kleinen Abstand von $30 \mathrm{~cm}$ die Anzahl der Eingriffe bis zur Erreichung der maximal zulässigen Personendosis vervielfacht werden kann [20]. Dieselbe Gruppe zeigt in dieser Untersuchung auch die Effektivität persönlicher Schutzausrüstung.

Im a.-p.-Strahlengang ist immer die Untertischposition der Strahlenquelle zu bevorzugen.

Eine weitere Möglichkeit, insbesondere in der Wirbelsäulenchirurgie Dosis einzusparen, kann die Computernavigation sein. In einigen eigenen Arbeiten konnten wir diesen Effekt bei der Platzierung von Pedikelschrauben nachweisen sowie eine Reduktion der Emission bei der Platzierung von sakroiliakalen Schrauben zeigen [21-23]. Dies konnte auch bei diversen weiteren Anwendungen von anderen Arbeitsgruppen bestätigt werden [24-27]. 


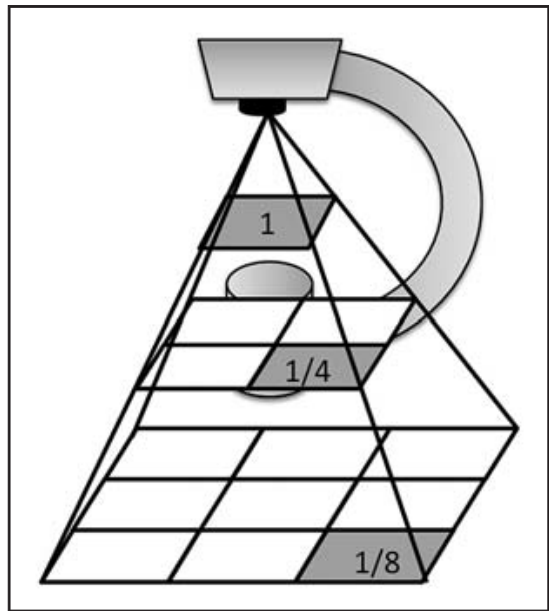

Abb. 1 Das Abstands-Quadrat-Gesetz besagt, dass sich die Strahlung mit dem Quadrat des Abstands zur Quelle verringert. Im Klartext heißt dies, dass sich die Strahlung bei doppeltem Abstand nicht nur halbiert, sondern sogar viertelt. Somit kommt also dem Abstand zur Quelle eine immense Bedeutung bei der persönlichen Exposition zu.

\section{Diskussion}

Das OP-Personal ist ionisierender Strahlung beinahe täglich ausgesetzt. Die dabei emittierte Strahlung ist ein Gesundheitsrisiko. Neben Hauterkrankungen können Schäden an der Augenlinse auftreten, es besteht ein Risiko, dass durch die Exposition eine maligne Erkrankung induziert wird. Der beste Schutz ist die Vermeidung von Strahlung, wenn dies nicht möglich ist, die Vermeidung der Exposition. Neben physikalischen Grunderkenntnissen konnte auch in den oben zitierten Studien nachgewiesen werden, dass auch unter den besonderen Bedingungen des Operationssaals optimalerweise eine hohe Distanz zur Strahlenquelle eingehalten werden soll. Dies wird auch stets von den Herstellern der Geräte gefordert. Jedoch ist jedem, der selber in diesem sensiblen Bereich arbeitet, klar, dass dies nicht in jeder Situation möglich ist. Neben der Einhaltung der Sterilität, die bei häufigem Abtreten vom Operationssitus definitiv in Gefahr ist, ist es dem OP-Team oft schlicht nicht möglich, Instrumente oder eine gerade reponierte Fraktur loszulassen und den Raum zu verlassen. Hier können die Erkenntnisse der o.g. Arbeiten jedoch helfen, die eigene Exposition auf ein Minimum zu reduzieren. An 1. Stelle zu nennen ist hier der Wert der persönlichen Schutzausrüstung. Neben der Schürze, welche die meisten Chirurgen tragen, ist die Verwendung eines Schilddrüsenschutzes keineswegs weniger wichtig. Gerade die Schilddrüse gilt als strahlen-
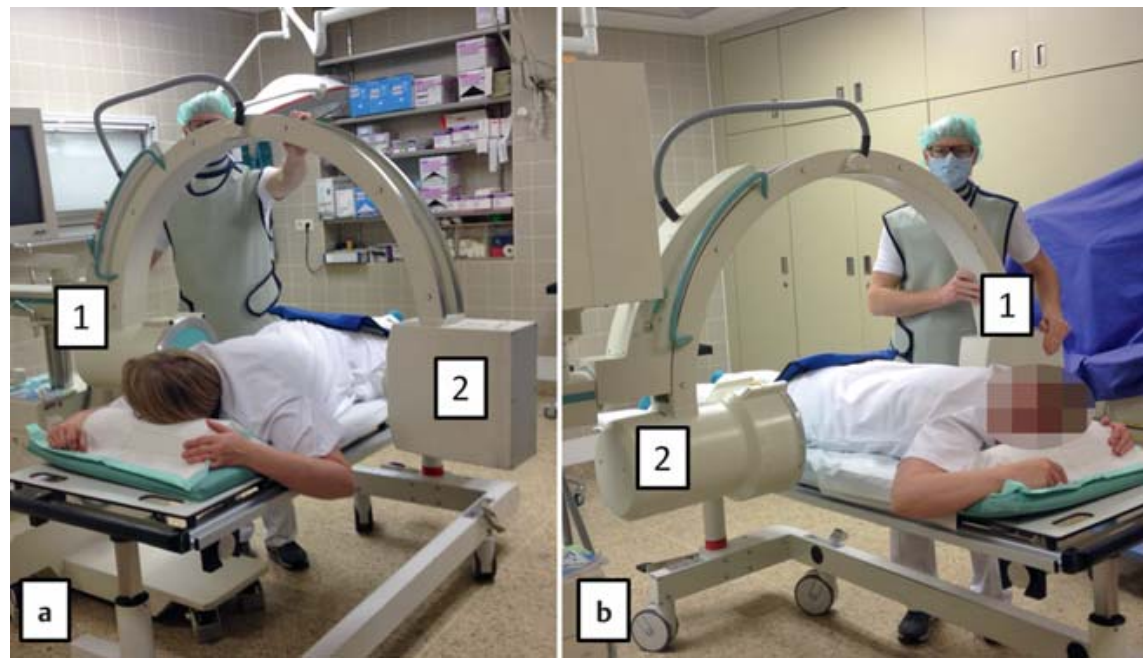

Abb. 2a und b Die seitliche Durchleuchtung ist ein wichtiger Bestandteil der intraoperativen Kontrolle. Mitarbeiter, die neben dem Empfänger (1) stehen (a), erhalten eine signifikant geringere Dosis als Personal, das neben der Strahlenquelle (2) positioniert ist (b). Daher sollten diese Personen möglichst vom Tisch abtreten, um eine hohe Exposition zu vermeiden.

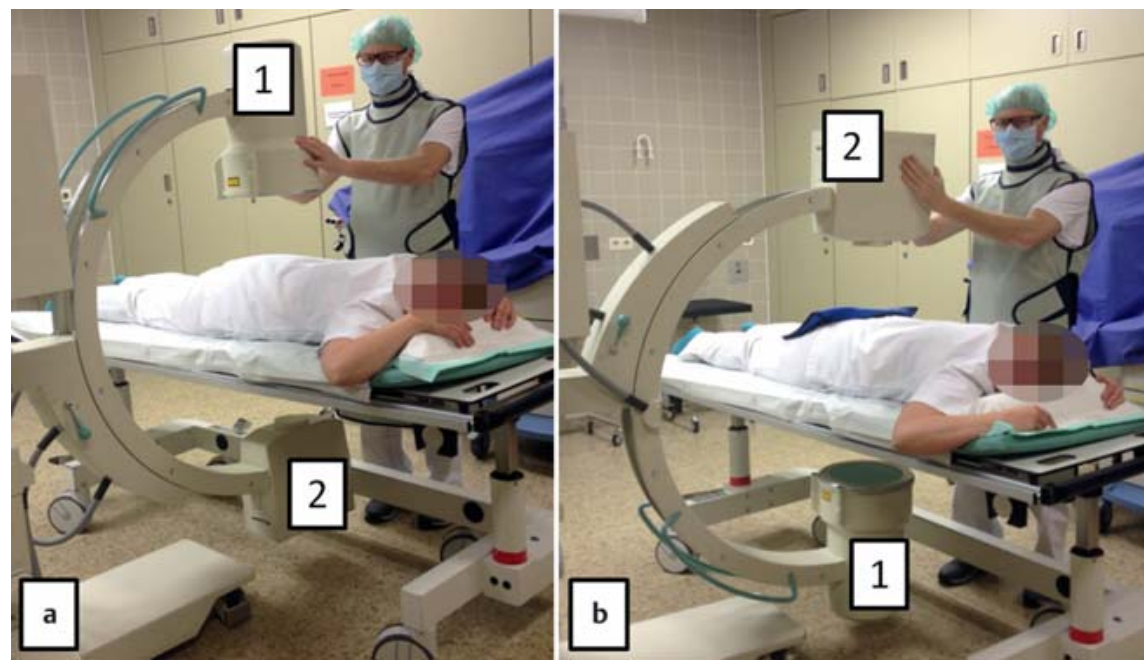

Abb. 3 a und $\mathbf{b}$ In aller Regel wird der C-Bogen wie in a abgebildet verwendet: der Bildverstärker ist über dem Tisch (1), die Quelle (2) der Strahlung ist unter dem Tisch positioniert. Gelegentlich wird der C-Bogen jedoch auch wie in b gezeigt verwendet, insbesondere dann, wenn mehr Arbeitsplatz benötigt wird, dies kann z. B. auch bei Infiltrationen an der Wirbelsäule erforderlich sein. In der letzteren Position ist mit einer deutlich erhöhten Exposition des Personals zu rechnen. Sie sollte daher, wenn möglich, vermieden werden.

sensibles Organ und sollte daher besonders beachtet werden. Auch die Position der Röntgenröhre ist entscheidend. Das Personal, das auf der Seite der Quelle steht, ist in besonders hohem Maße gefährdet (Abb. 2). Daher sollte es diesen Mitarbeitern ermöglicht werden, bei Durchleuchtung vom Tisch abzutreten und den weitestmöglichen Sicherheitsabstand einzuhalten. Die meisten Chirurgen verwenden die Untertischposition. Dabei wird der Großteil der Strahlung vom Tisch und auch vom Patienten reflektiert und zurück Richtung Boden geworfen. Wesentlich gefährlicher ist die Positionierung der Röhre über dem Patienten, da hierbei die Streustrahlung von Tisch und Patient direkt auf das OPTeam zurückreflektiert wird (Abb.3). Dieses Problem kann möglicherweise durch neue Flachdetektorbildwandler gelöst werden, die ebenso eine verbesserte Arbeitshöhe in Untertischposition versprechen, da die Detektoreinheit deutlich platzsparender ist (Abb. 4).

Die kumulative Erfahrung des Operateurs und des 1. Assistenten beeinflussen die Emission von Strahlung signifikant. 


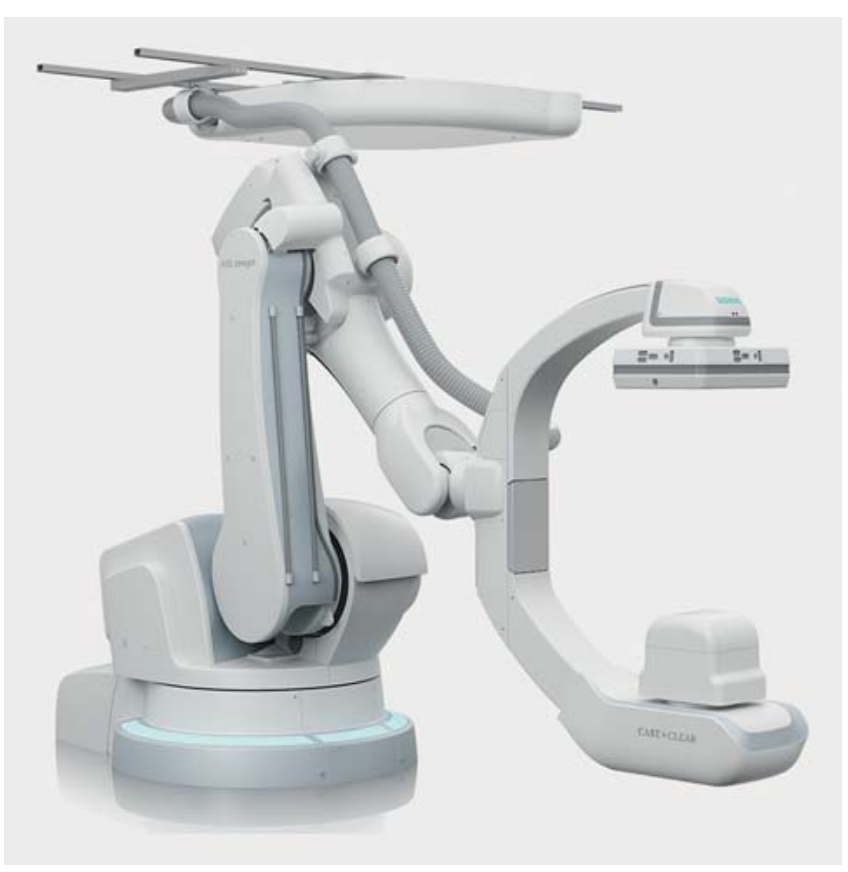

Abb. 4 Flachdetektorbildwandler haben eine verbesserte $\mathrm{Ar}$ beitshöhe, da der Bildverstärker deutlich weniger raumfordernd ist als bei herkömmlichen C-Bögen (hier: Artis zeego ${ }^{\circledR}$, Siemens, Erlangen).

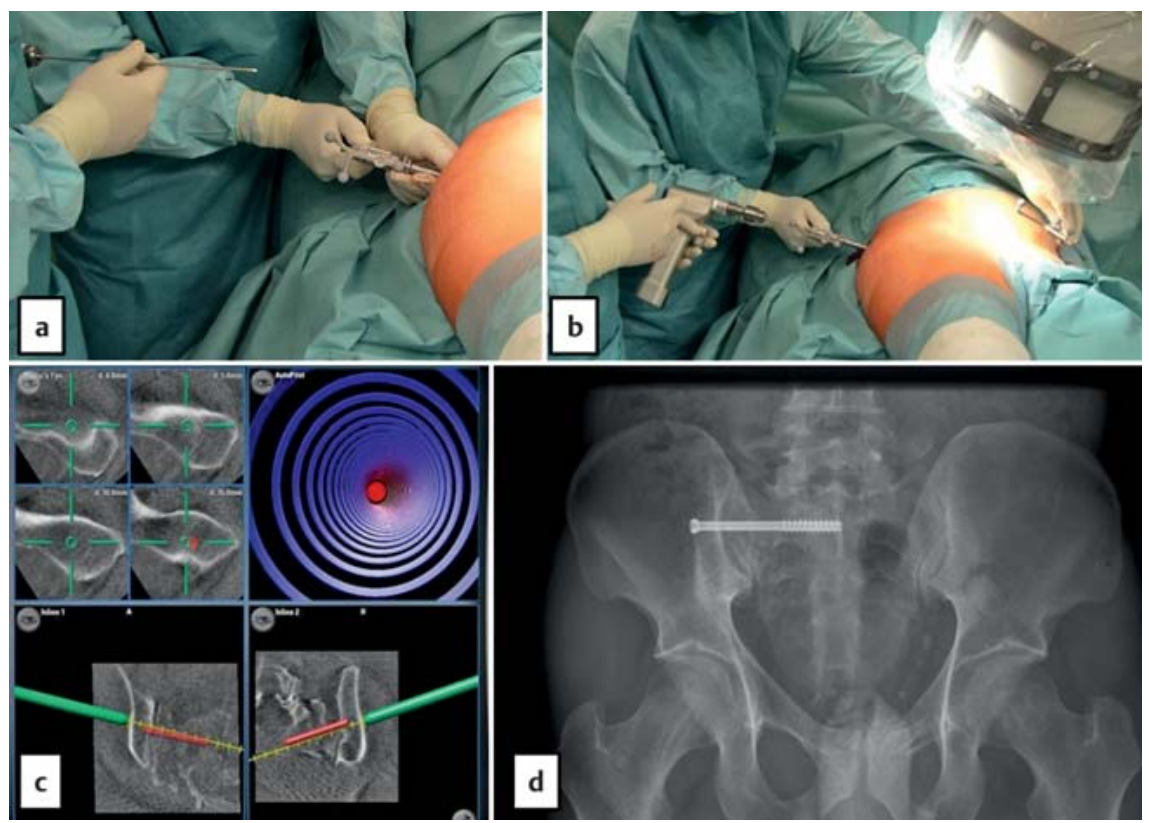

Abb. 5 a bis d Navigierte Verfahren können ebenso die Emission von Strahlung vermindern. Beim Einbringen einer Schraube iliosakral wird eine navigierte Bohrhülse verwendet (a). Nun kann darüber ein Kirschner-Draht navigiert eingebracht werden (b). Das System erlaubt dabei nach Einspielen der Bilder röntgenfrei die Kontrolle des Drahtes oder des zu navigierenden Instruments in Echtzeit (c). In der postoperativen Kontrolle zeigt sich eine korrekt sitzende Schraube (d).

In unserer Studie an der Strahlenemission bei Radiusfrakturen konnte ein weiterer, sehr interessanter Aspekt hervorgehoben werden. Es gibt bestimmte Faktoren, die bereits präoperativ darauf hindeuten, dass evtl. eine höhere Dosis zum Einsatz kommen wird. Neben der Schwere der Fraktur ist hier insbesondere die Teamerfahrung entscheidend. Die Kombination junger Assistent, jun- ger Oberarzt und eine Typ-C3-Fraktur können also darauf hinweisen, dass hier der persönlichen Schutzausrüstung eine noch höhere Bedeutung zukommt. Es empfiehlt sich also, dann z. B. die dickere Schürze zu verwenden, evtl. auch ein komplett geschlossenes Modell, ebenso sollte ein Schilddrüsenschutz zum Einsatz kommen. Je nach operativem Vorgehen können auch Bleiglaswände oder
Tischanbauten die Strahlenexposition deutlich vermindern. Wenig verwendet werden auch Schutzbrillen zur Vermeidung von Augenerkrankungen, hierzu fehlen allerdings auch richtungsweisende Studien.

Der beste Schutz vor Strahlung ist der Verzicht darauf. Wenn Röntgen erforderlich ist, sollte ein größtmöglicher Abstand zur Quelle bestehen. Optimale Schutzausrüstung ist im OP beim Einsatz eines Bildverstärkers unerlässlich.

Neuere OP-Techniken bedeuten nicht zwangsläufig eine höhere Emission von Strahlung. So ist bei minimalinvasiven Eingriffen die Exposition zwar höher, durch navigierte Verfahren kann dieser Effekt eventuell wieder minimiert werden (Abb. 5).

Ein neuer Ansatz in der besseren Ausbildung und Schulung von Ärzten und weiteren Anwendern bieten Lernplattformen, die ein zeit- und ortsungebundenes Lernen und Weiterbilden erlauben. Einige interessante Ansätze wurden durch unsere Arbeitsgruppe mithilfe der AO-Foundation verwirklicht (https://aotrauma. aofoundation.org/Structure/education/ self-directed-learning/online-learning/ elearning-modules/Pages/elearningmodules.aspx\#null) (Abb. 6 und 7) [28].

Zusammengefasst bedarf es sicherlich weiterer Anstrengungen, um die Bedeutung des Strahlenschutzes im OP herauszuheben und zu verbessern. Gefordert werden sollte neben den eher theoretisch ausgerichteten, vorgeschriebenen Kursen auch praktische Übungen, gerade für das Personal, das den C-Bogen dann auch bedienen soll. Röntgentechniker, die speziell für den Umgang mit diesen Geräten ausgebildet sind und in englischsprachigen Ländern eingesetzt werden, wären wünschenswert, jedoch bei einer entsprechenden Ausbildung des sich im Einsatz befindlichen OP-Personals wahrscheinlich nicht erforderlich, insbesondere unter Beachtung ökonomischer Faktoren. Unverändert sollte jedwede Strahlung immer nach dem ALARA-Prinzip erfolgen: As Low As Reasonably Achievable, also immer nur so viel an Strahlung einzusetzen, die zur Erreichung des operativen Zieles unbedingt erforderlich ist.

ALARA = As Low As Reasonably Achievable. Also: Nur so viel Strahlung wie unbedingt erforderlich zum Erreichen des Zieles einsetzen! 


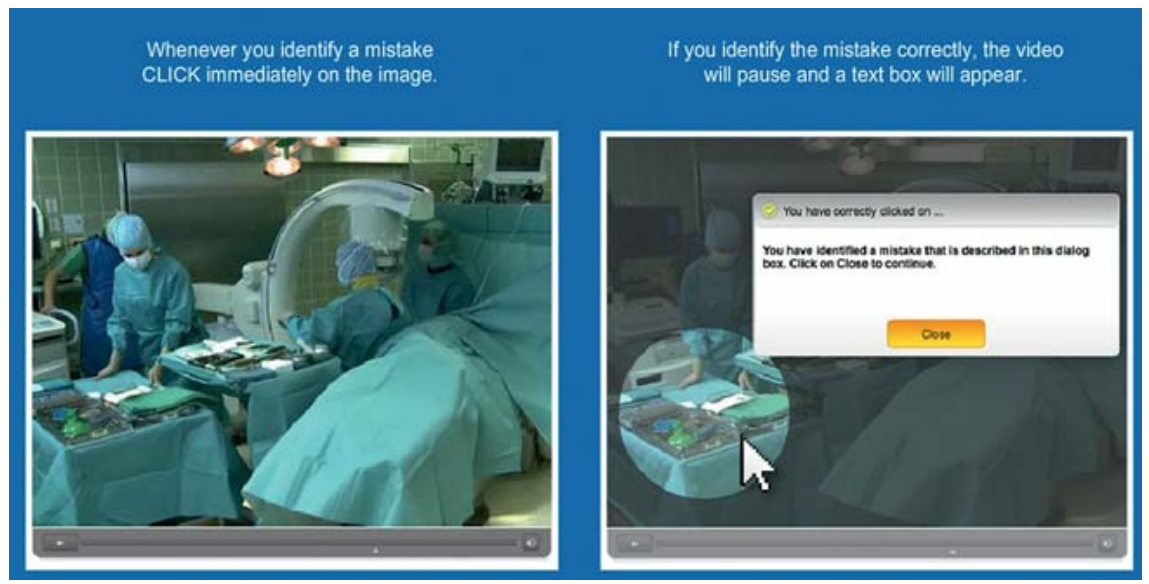

Abb. 6 In einem bis dato neuartigen E-Learning-Modul wird in einem interaktiven Video auf mögliche Fehlerquellen anhand klinischer Fälle hingewiesen. Der Benutzer kann dabei entdeckte Fehler anklicken und bekommt am Ende eine Auswertung sowie weiterführende Quellen genannt.

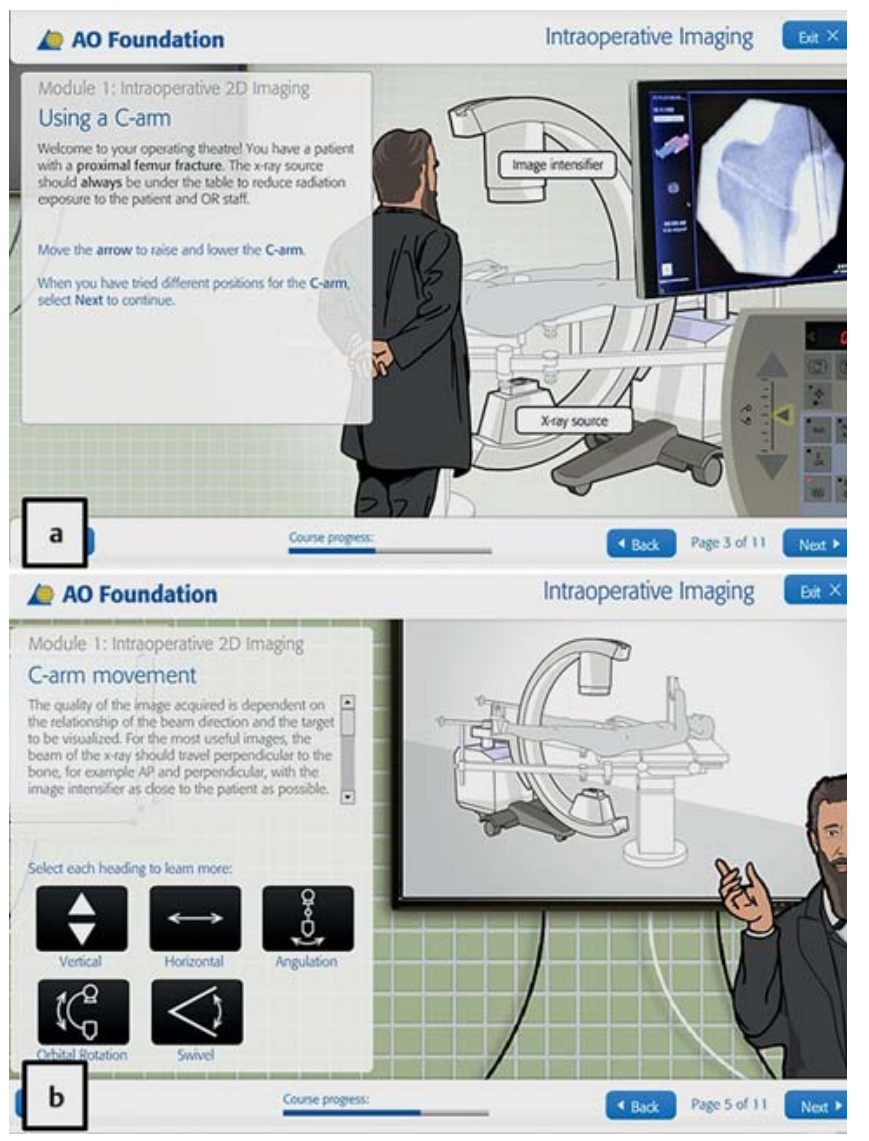

Abb. $7 \mathbf{a}$ und $\mathbf{b}$ In einem weiteren EL-earning-Modul werden Basisinhalte interaktiv vermittelt (a). So kann der Benutzer neben gängigen Bedienelementen an mobilen Bildverstärkern (b) auch aktiv einen 2-D- sowie einen 3-D-C-Bogen steuern und sieht unmittelbar, welche Ergebnisse eine Änderung des ObjektBildverstärker-Abstands oder die Anwendung der Blende hat.

\section{Literatur}

${ }^{1}$ Singer G. Occupational radiation exposure to the surgeon. J Am Acad Orthop Surg 2005; 13: 69-76

2 Mastrangelo G, Fedeli U, Fadda $E$ et al. Increased cancer risk among surgeons in an orthopaedic hospital. Occup Med (Lond) 2005; 55: 498-500

${ }^{3}$ Yu E, Khan SN. Does less invasive spine surgery result in increased radiation exposure? A systematic review. Clin Orthop Relat Res 2014; 472: 1738-1748
${ }^{4}$ Wrixon $A D$. New ICRP recommendations. J Radiol Prot 2008; 28: 161-168

5 Wrixon $A D$. New recommendations from the International Commission on Radiological Protection-a review. Phys Med Biol 2008; 53: R41-R60

${ }^{6}$ Blakely EA. Biological effects of cosmic radiation: deterministic and stochastic. Health Phys 2000; 79: 495-506

7 Mettler FA jr., Koenig TR, Wagner LK et al. Radiation injuries after fluoroscopic procedures. Semin Ultrasound CT MR 2002; 23 : 428-442
${ }^{8}$ Koenig TR, Mettler FA, Wagner LK. Skin injuries from fluoroscopically guided procedures: part 2, review of 73 cases and recommendations for minimizing dose delivered to patient. AJR Am J Roentgenol 2001; 177: $13-$ 20

${ }^{9}$ Koenig TR, Wolff D, Mettler FA et al. Skin injuries from fluoroscopically guided procedures: part 1, characteristics of radiation injury. AJR Am J Roentgenol 2001; 177: 3-11

10 Williams D. Radiation carcinogenesis: lessons from Chernobyl. Oncogene. 2008; 27 (Suppl. 2): S9-S18

${ }^{11}$ Dresing K. [X-ray in trauma and orthopedic surgery. Physical and biological impact, reasonable use, and radiation protection in the operating room]. Oper Orthop Traumatol 2011; 23: 70-78

${ }^{12}$ Muller LP, Suffner J, Wenda K et al. [Radiation burden to the hands of surgeons in intramedullary nailing]. Unfallchirurgie 1996; 22: 53-259

${ }^{13}$ Barry TP. Radiation exposure to an orthopedic surgeon. Clin Orthop Relat Res 1984; 182: $160-164$

14 Sanders R, Koval KJ, DiPasquale T et al. Exposure of the orthopaedic surgeon to radiation. J Bone Joint Surg Am 1993; 75: 326-330

15 Kraus M, Roderer G, Max M et al. Influence of fracture type and surgeon experience on the emission of radiation in distal radius fractures. Arch Orthop Trauma Surg 2013; 133: 941-946

${ }^{16}$ Oddy MJ, Aldam $\mathrm{CH}$. Ionising radiation exposure to orthopaedic trainees: the effect of sub-specialty training. Ann R Coll Surg Engl 2006; 88: 297-301

17 Rampersaud YR, Foley KT, Shen AC et al. Radiation exposure to the spine surgeon during fluoroscopically assisted pedicle screw insertion. Spine (Phila Pa 1976) 2000; 25: $2637-$ 2645

${ }^{18}$ Lee K, Lee KM, Park MS et al. Measurements of surgeons' exposure to ionizing radiation dose during intraoperative use of $\mathrm{C}$-arm fluoroscopy. Spine (Phila Pa 1976) 2012; 37: 1240 1244

${ }^{19}$ Jones DP, Robertson PA, Lunt B et al. Radiation exposure during fluoroscopically assisted pedicle screw insertion in the lumbar spine. Spine (Phila Pa 1976) 2000; 25: 1538-1541

20 Mehlman CT, DiPasquale TG. Radiation exposure to the orthopaedic surgical team during fluoroscopy: "how far away is far enough?". J Orthop Trauma 1997; 11: 392-398

${ }^{21}$ Kraus MD, Krischak G, Keppler $P$ et al. Can computer-assisted surgery reduce the effective dose for spinal fusion and sacroiliac screw insertion? Clin Orthop Relat Res 2010; 468: 2419-2429

22 Gebhard FT, Kraus MD, Schneider E et al. Does computer-assisted spine surgery reduce intraoperative radiation doses? Spine (Phila Pa 1976) 2006; 31: 2024-2027

${ }^{23}$ Gebhard F, Kraus M, Schneider E et al. [Radiation dosage in orthopedics - a comparison of computer-assisted procedures]. Unfallchirurg 2003; 106: 492-497

${ }^{24}$ Kim CW, Lee YP, Taylor W et al. Use of navigation-assisted fluoroscopy to decrease radiation exposure during minimally invasive spine surgery. Spine J 2008; 8: 584-590

25 Slomczykowski M, Roberto M, Schneeberger P et al. Radiation dose for pedicle screw insertion. Fluoroscopic method versus computer-assisted surgery. Spine (Phila Pa 1976) 1999; 24: 975-982

${ }^{26}$ Smith HE, Welsch MD, Sasso RC et al. Comparison of radiation exposure in lumbar pedicle screw placement with fluoroscopy vs. computer-assisted image guidance with intraop- 
erative three-dimensional imaging. J Spinal Cord Med 2008; 31: 532-537

27 Zwingmann J, Konrad G, Kotter E et al. Computer-navigated iliosacral screw insertion reduces malposition rate and radiation exposure. Clin Orthop Relat Res 2009; 467: $1833-$ 1838

${ }^{28}$ Kraus $M$, Gebhard F. AO Trauma eLearning Modules. 2013. Davos, CH, AO Trauma. 10-62014. Im Internet: https://aotrauma. aofoundation.org/Structure/education/selfdirected-learning/online-learning/elearningmodules/Pages/elearning-modules. aspx\#null; Stand: 05.11.2014
PD Dr. med. Michael Kraus

Oberarzt

Klinik für Orthopädie, Unfallchirurgie und Wirbelsäulentherapie

Donau-Ries Klinik Donauwörth

Neudegger Allee 6

86609 Donauwörth

Deutschland

Tel.: 0906/7821011

Fax: 0906/7821040

kraus@email.de

\section{Michael Redies}

Global Education Manager

AO Trauma

Clavadelerstrasse 8

7270 Davos

Schweiz

\section{Dr. med. Peter Richter}

Assistenzarzt

Klinik für Unfall-, Hand-, Plastische und Wiederherstellungschirurgie

Universitätsklinikum Ulm

Albert-Einstein-Allee 23

$89081 \mathrm{Ulm}$

Deutschland 